\title{
Mortalidad infantil por malformaciones congénitas en la Ciudad Autónoma de Buenos Aires (1998-2015): Análisis espacial, temporal y relación con la condición socioeconómica
}

Infant mortality due to congenital malformations in the Autonomous City of Buenos Aires (1998-2015): Spatial, temporal analysis and relation to the socioeconomic status

\author{
Dr. Rubén A. Bronberga y Dr. José E. Dipierri ${ }^{b}$
}

\section{RESUMEN}

Introducción. En Argentina, las malformaciones congénitas (MC) representan la segunda causa de muerte en menores de 1 año.

Objetivo. Analizar la variación temporaly espacial de la mortalidad infantil por MC en la Ciudad Autónoma de Buenos Aires y su relación con un indicador de desarrollo socioeconómico.

Materiales y método. Los datos de nacimientos y muertes de menores de 1 año deedad codificados con la Clasificación Internacional de Enfermedades (CIE-10) provinieron del Ministerio de Salud. Para las zonas geográficas (Norte, Centro y Sur), sistemas (nervioso, cardiovascular y anomalías cromosómicas) y 28 malformaciones específicas, se estimaron la tasa de mortalidad infantil por MC(TMI-MC) y el porcentaje de muertes por MC (PM-MC) en 3 períodos (1998-2003, 2004-2009,

a. Área de Genética Médica y Poblacional, Sección Neonatología, Departamento Maternoinfantojuvenil, Hospital General de Agudos Dr. J. M. Ramos Mejía, Ciudad de Buenos Aires.

b. Instituto de Biología de la Altura,

Universidad Nacional de Jujuy, San Salvador de Jujuy.

Correspondencia: Dr. Rubén A. Bronberg: rabronberg@intramed.net

\section{Financiamiento: \\ Ninguno.}

Conflicto de intereses: Ninguno que declarar.

Recibido: 22-7-2018 Aceptado: 18-12-2018 2010-2015). La tendencia secular y el riesgo de muerte se estimaron con un modelo de regresión dePoisson. Mediante el análisis de componentes principales, se definió un indicador de desarrollo socioeconómico que se correlacionó con la TMIMC y el PM-MC.

Resultados. LaTMI-MCy elPM-MCpresentaron, respectivamente, unatendenciasecularnegativay positiva con significación estadística y exhibieron una diferenciación por zonas. La TMI-MC descendió para las malformaciones del sistema nervioso central y cardiovascular, y aumentó en las anomalías cromosómicas ( $\mathrm{p}<0,05)$. La TMIMC y el PM-MC se correlacionaron de modo positivo y negativo, respectivamente, con el indicador socioeconómico ( $\mathrm{p}<0,05)$.

Conclusión. Los indicadores de mortalidad infantil por MC de la Ciudad Autónoma de Buenos Aires exhiben una heterogeneidad espacial y temporal, y se relacionan con las características socioeconómicas zonales.

Palabras clave: mortalidad infantil, anomalías congénitas.

http: / / dx.doi.org/10.5546/aap.2019.171

Texto completo en inglés:

http:/ / dx.doi.org/10.5546/aap.2019.eng.171

Cómo citar: Bronberg RA. Dipierri JE. Mortalidad infantil por malformaciones congénitas en la Ciudad Autónoma de Buenos Aires (1998-2015): Análisis espacial, temporal y relación con la condición socioeconómica. Arch Argent Pediatr 2019;117(3):171-178.

\section{GLOSARIO}

ACP: Análisis de componentes principales.

CABA: Ciudad Autónoma de Buenos Aires.

MC: Malformaciones congénitas.

MI: Mortalidad infantil.

PM-MC: Proporción de muertes por MC.

TMI-MC: Tasa de mortalidad infantil por MC.

TS: Tendencia secular.

\section{INTRODUCCIÓN}

En la mayoría de los países de América Latina, las muertes en los menores de un año por malformaciones congénitas (MC) ocupan entre el segundo y el quinto lugar, y contribuyen, entre el $2 \%$ y el $27 \%$, a la mortalidad infantil (MI) total. ${ }^{1}$

En las últimas décadas, en países con distinto grado de desarrollo, se ha observado un patrón caracterizado por una disminución de la MI total, por el control de las enfermedades infecciosas y las deficiencias nutricionales, que se acompaña de una reducción de la tasa de MI por MC (TMI-MC) y un aumento del porcentaje de muertes infantiles por $\mathrm{MC}(\mathrm{PM}-\mathrm{MC}) .^{2-6}$

De acuerdo con Rosano et al. (2000), ${ }^{2}$ la TMI-MC es más alta en los países más pobres, y el PM-MC, más alto en los más desarrollados. En Argentina, las poblaciones con menor PM-MC, menos desarrolladas y con mayores porcentajes de necesidades 
básicas insatisfechas se sitúan en el norte del país. ${ }^{7}$

La Ciudad Autónoma de Buenos Aires (CABA) ocupa la parte del territorio argentino con el índice de desarrollo humano (IDH) más alto $(0,889)$ y presenta los porcentajes de población con cobertura pública (el 17,7\%), con necesidades básicas insatisfechas (el 7,0 \%) y analfabetismo (el 0,5\%) más bajos del país. ${ }^{8}$

Las estructuras y dinámicas urbanas actuales conducen, en las grandes ciudades, a transformaciones socioterritoriales que se manifiestan por desigualdades y microdiferenciaciones territoriales y discontinuidades espaciales. ${ }^{9}$ La CABA no escapa a este fenómeno y se detecta en ella una marcada segregación socioterritorial, ${ }^{10}$ demográfica, ${ }^{11}$ demogenética ${ }^{12}$ y un crecimiento metropolitano mixto (dispersión/policentrismo). ${ }^{13}$ Esta diferenciación espacial también se manifiesta en los indicadores de salud. ${ }^{14,15}$

El objetivo del trabajo fue analizar la variación temporal y espacial de la TMI-MC y del PM-MC en la CABA, y su relación con un indicador de desarrollo socioeconómico.

\section{MATERIALES Y MÉTODO}

Estudio poblacional basado en datos de nacimientos y muertes infantiles sucedidos en 1998-2015 recabados por la Dirección de Estadística e Información de Salud (DEIS) del Ministerio de Salud de Argentina. ${ }^{16}$

Se recopiló y analizó información no vinculable de fuentes secundarias de conocimiento público con el correspondiente resguardo del secreto estadístico, por lo cual no requirió consentimiento.

Según el lugar de residencia materna, se utilizaron las siguientes variables: a) número de fallecidos durante el primer año de vida; b) número de nacidos vivos; c) causa de las defunciones por MC codificadas según la Clasificación Internacional de Enfermedades, décima revisión ${ }^{17}$ (CIE-10) (Códigos Q00-Q99).

Se calcularon, independientemente del sexo, la TMI-MC (número de defunciones infantiles por MC / 1000 recién nacidos vivos $-\mathrm{RNV}-$ ) y el PM-MC (número de defunciones infantiles por MC/ 100 fallecidos) para toda la CABA, las 28 circunscripciones electorales y 15 comunas distribuidas en 3 zonas geográficas (Norte, Centro y Sur) definidas por la Dirección General de Estadística y Censos de la CABA.

Debido a las modificaciones de la fuente de datos utilizada, el registro de las muertes se realizó por circunscripciones electorales y por comunas en diferentes años. Las zonas geográficas se conformaron desde 1998 a 2010 con las circunscripciones electorales: a) Norte 16, 17, 18, 19; b) Centro 5, 6, 7, 8, 9, 10, 11, 12, 13, $14,15,20,25,26,27,28$; c) Sur 1, 2, 3, 4, 22, 23, 21, 24. Desde 2011 a 2015, se conformaron con las comunas: a) Norte 2, 13, 14; b) Centro 3, 5, 6, 7, 11, 12, 15; c) Sur 4, 8, 9, 10.

Los intervalos de confianza de la TMI-MC y el PM-MC se calcularon según la distribución de Poisson, apropiada para eventos raros.

De acuerdo con el modelo matemático aplicado, el período total se dividió en tres subgrupos: 1998-2003, 2004-2009 y 2010-2015. La tendencia secular (TS) para todo el período y la variación del riesgo de muerte para el subperíodo 2010-2015 en relación con el basal (1998-2003) se estimaron mediante modelos de regresión de Poisson, utilizando a los fallecidos durante el primer año de vida por MC como variable dependiente, las unidades de tiempo como variables independientes y a los fallecidos totales o a los recién nacidos como población de referencia. Para ello, se empleó el paquete estadístico Stata 12.1.

Se calculó la variación temporal de la TMIMC y el PM-MC para la CABA y / o las zonas geográficas de 28 malformaciones específicas de acuerdo con los siguientes subgrupos: a) sistema nervioso central (SNC, Q00-Q079); b) sistema cardiovascular (SCV, Q200-Q289); c) anomalías cromosómicas (Q900-Q999).

Para determinar un único factor que resumiera el efecto de factores socioeconómicos y demográficos, se utilizó el análisis de componentes principales (ACP) recurriendo a los valores porcentuales de las siguientes variables estandarizadas en cuanto a las comunas (datos de 2011 a 2015): a) población que utilizaba solo sistema público médico de salud; b) población que utilizaba solo sistema prepago médico de salud; c) población de alumnos con fracaso escolar; d) población con ingresos inferiores a la canasta total; e) población bajo la línea de la pobreza, f) población con necesidades básicas insatisfechas; g) viviendas precarias; h) individuos desocupados (Dirección General de Estadística y Censos de la CABA)..$^{18}$ Se aplicó la prueba de Pearson para correlacionar el primer componente del ACP con la TMI-MC y el PM-MC.

\section{RESULTADOS}

La TMI-MC tendió a disminuir; lo contrario sucedió con el PM-MC, que, claramente, aumentó 
en las 3 zonas geográficas (Tabla 1, Figura 1).

La TMI-MC para toda la ciudad, para los tres subperíodos, fue del 2,89, del 2,45 y del $2,55 \%$ con una TS, para todo el período, significativamente negativa $(-0,009)$. Por cada año en la CABA, la TMI-MC descendió el 0,9\%. Si se compara el subperíodo 2010-2015 con el subperíodo basal 1998-2003, el riesgo de morir por una MC en el primer año de vida descendió significativamente un $12 \%$. En cuanto a las zonas, la TMI-MC presentó una TS negativa no significativa. En los 3 períodos, la TMI-MC más alta se presentó en la zona Sur, seguida por la zona Centro y la Norte (Tabla 1, Figuras 1 y 2).

FIGURA 1. Tasa de mortalidad infantil por malformaciones congénitas y porcentaje de muertos por malformaciones congénitas de la Ciudad Autónoma de Buenos Aires y zonas geográficas de todo el período

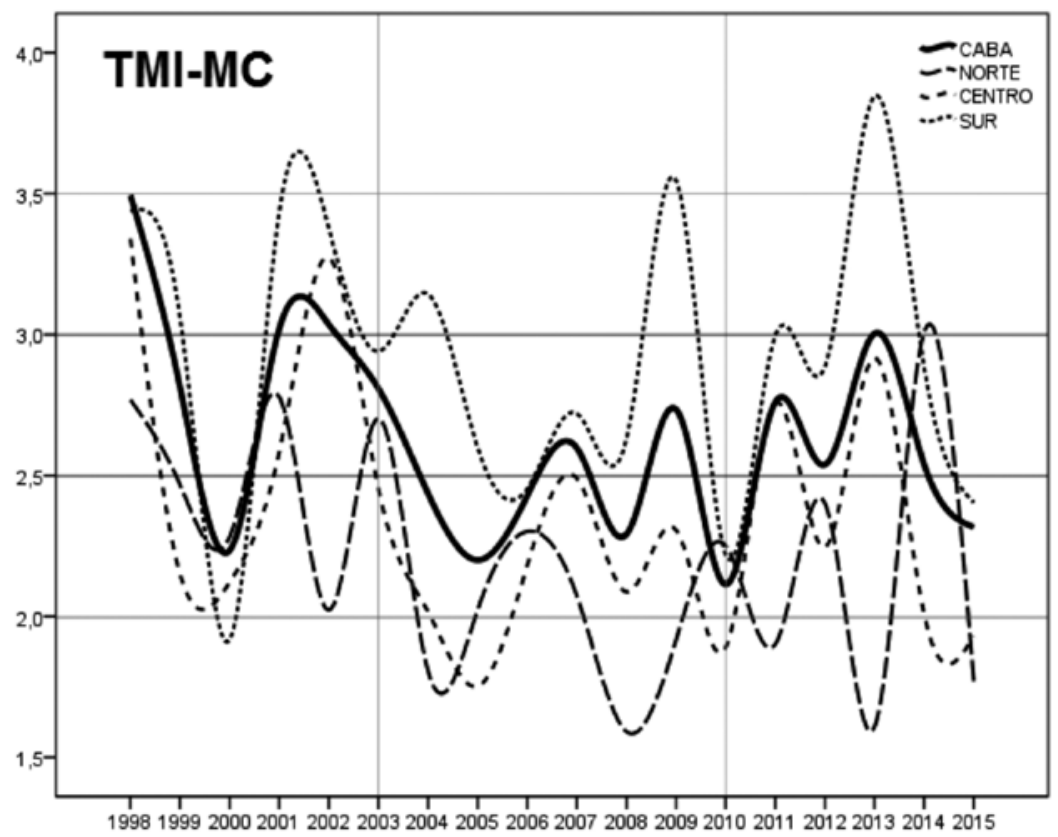

TMI-MC: tasa de mortalidad infantil por malformaciones congénitas; CABA: Ciudad Autónoma de Buenos Aires.

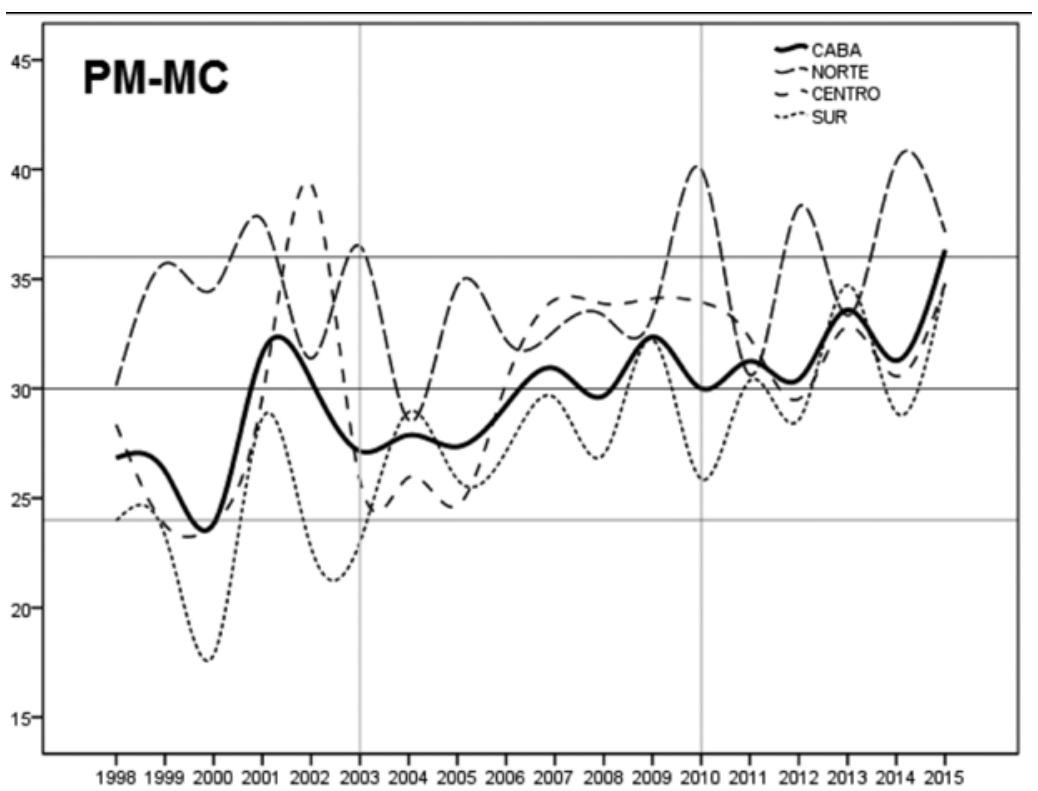

PM-MC: porcentaje de muertos por malformaciones congénitas. 
El PM-MC para toda la ciudad en los tres subperíodos fue del $27,6 \%$, del $29,6 \%$ y del $32,0 \%$. La TS para todo el período (1998-2015) fue significativamente positiva $(0,014)$. Por cada año, el PM-MC aumentó un 1,4\%. Si se compara el subperíodo 2010-2015 con el subperíodo basal 1998-2003, el peso relativo de la MC como causa de la MI aumentó significativamente un $16 \%$. En cuanto a las zonas geográficas, el PM-MC presentó una TS positiva, significativa en las regiones Centro y Sur. En los 3 períodos, el PM-MC más alto se presentó en la zona Norte, seguida por la zona Centro y la Sur (Tabla 1, Figura 1).

Por cada año en la CABA, las TMI-MC específicas para malformaciones del SNC y del SCV descendieron el 3,8\% y el $2,3 \%$,

TABLA 1. Tasa de mortalidad infantil por malformaciones congénitas y proporción de muertos por malformaciones congénitas, por subperiodos, tendencia secular de todo el período y riesgo del último perído (2010-2015) en relación con el basal (1998-2003) de la Ciudad Autónoma de Buenos Aires y regiones geográficas

\begin{tabular}{|c|c|c|c|c|c|}
\hline \multirow[t]{2}{*}{ CABA/regiones } & \multicolumn{3}{|c|}{$\begin{array}{l}\text { TMI-MC } \\
\text { (IC } 95 \%)\end{array}$} & \multirow[t]{2}{*}{ TS } & \multirow[t]{2}{*}{ Riesgo } \\
\hline & 1998-2003 & 2004-2009 & 2010-2015 & & \\
\hline CABA & $2,89(2,57-3,21)$ & $2,45(2,16-2,74)$ & $2,55(2,25-2,84)$ & $-0,009^{*}$ & $0,88^{*}$ \\
\hline Norte & $2,51(1,83-3,18)$ & $1,95(1,35-2,55)$ & $2,16(1,51-2,81)$ & $-0,012$ & 0,86 \\
\hline Centro & $2,64(2,18-3,11)$ & $2,13(1,73-2,54)$ & $2,30(1,90-2,71)$ & $-0,010$ & 0,87 \\
\hline \multirow[t]{2}{*}{ Sur } & $3,02(2,43-3,62)$ & $2,85(2,33-3,38)$ & $2,85(2,29-3,41)$ & $-0,002$ & 0,94 \\
\hline & & PM-MC & & & \\
\hline CABA & $27,6(24,5-30,7)$ & $29,6(26,1-33,1)$ & $32,0(28,3-35,7)$ & $0,014^{*}$ & $1,16^{*}$ \\
\hline Norte & $34,3(25,0-43,5)$ & $32,3(22,4-42,2)$ & $36,8(25,7-47,9)$ & 0,007 & 1,07 \\
\hline Centro & $28,2(23,3-33,2)$ & $30,3(24,5-36,0)$ & $32,1(26,5-37,7)$ & $0,013^{*}$ & 1,14 \\
\hline Sur & $23,3(18,8-27,9)$ & $28,6(23,3-33,8)$ & $30,3(24,3-36,3)$ & $0,022^{*}$ & $1,30^{*}$ \\
\hline
\end{tabular}

* Nivel de significación $p<0,05$; IC 95: intervalo de confianza del 95 \%; TMI-MC: tasa de mortalidad infantil por malformaciones congénitas; PM-MC: porcentaje de muertos por malformaciones congénitas; TS: tendencia secular; CABA: Ciudad Autónoma de Buenos Aires.

FIGURA 2. Zonas geográficas (Norte, Centro y Sur), subdivididas por circunscripciones electorales coloreadas por cuartiles de la tasa de mortalidad infantil por malformaciones congénitas

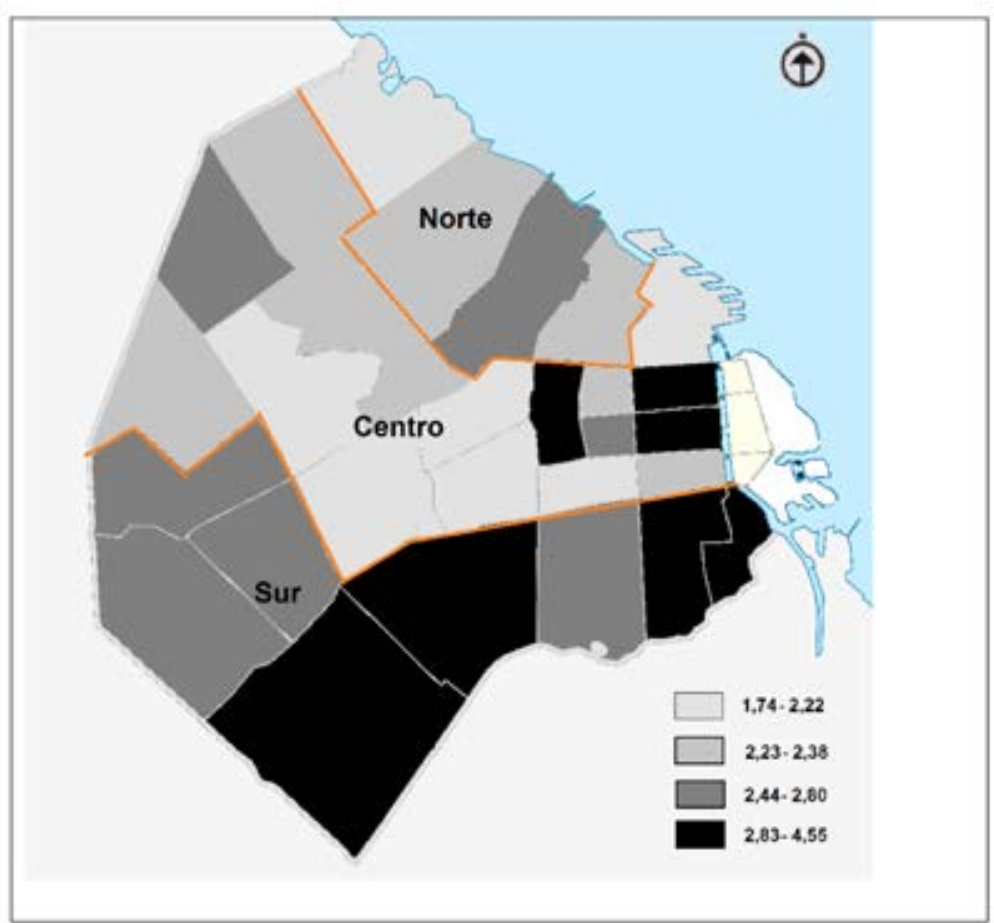


respectivamente, mientras que la TMI-MC para anomalías cromosómicas se incrementó un 3,9\% (Tabla 2). Si se compara el subperíodo 2010-2015 con el subperíodo basal 1998-2003, el riesgo de morir durante el primer año de vida por malformaciones del SNC y del SCV descendió un $38 \%$ y un $25 \%$, respectivamente, y aumentó para las anomalías cromosómicas un $49 \%$. El PMMC por sistemas presentó un comportamiento semejante a la TMI-MC (Tabla 2). Si se compara el subperíodo 2010-2015 con el subperíodo basal 1998-2003, el peso relativo de la MC como causa de la MI de malformaciones del SNC y del SCV disminuyó un $30 \%$ y un $15 \%$, respectivamente, y aumentó para las anomalías cromosómicas un $70 \%$.

Las TMI-MC específicas para anencefalia y espina bífida presentaron una TS negativa y una disminución del riesgo estadísticamente significativa. En cambio, el conducto arterioso permeable, la holoprosencefalia y la trisomía 18 presentaron una TS positiva y mayor riesgo estadísticamente significativo (Tabla 3).

El primer componente del ACP que explica el $72,8 \%$ de la varianza $-0,946 \times(a)-0,798 \times(b)+$ $0,735 \times(\mathrm{c})+0,933 \times(\mathrm{d})+0,960 \times(\mathrm{e})+0,910 \times(\mathrm{f})$ $+0,653 \times(\mathrm{g})+0,839 \times(\mathrm{h})$ - indica que, a mayor valor de este, menor desarrollo socioeconómico y muestra una clara diferenciación por zonas: se presenta el valor más alto en la zona Sur (108), seguido por la zona Centro $(64,1)$ y Norte $(16,3)$. Para toda la CABA, se observó una correlación positiva y significativa $(\mathrm{r}=0,716 ; \mathrm{p}<0,002)$ del primer componente del ACP con la TMI-MC y una correlación negativa significativa con el PM$\mathrm{MC}(\mathrm{r}=-0,545 ; \mathrm{p}<0,036)$ (Figura 3). En cuanto a las zonas, las correlaciones de la TMI-MC y el PM$\mathrm{MC}$ con el primer componente fueron del mismo signo que para toda la $\mathrm{CABA}$, pero no fueron estadísticamente significativas.

TABLA 2. Tasa de mortalidad infantil por malformaciones congénitas y proporción de muertos por malformaciones congénitas, por sistemas según subperíodos, tendencia secular y riesgo del último perído (2010-2015) en relación con el basal (1998-2003) de la Ciudad Autónoma de Buenos Aires y zonas geográficas

\begin{tabular}{|c|c|c|c|c|c|c|}
\hline \multirow[t]{2}{*}{ Sistema } & \multirow[t]{2}{*}{$\begin{array}{l}\text { CABA } \\
\text { Zonas }\end{array}$} & \multicolumn{4}{|c|}{$\begin{array}{c}\text { TMI-MC (10 }) \\
\text { (IC } 95 \%)\end{array}$} & \multirow[b]{2}{*}{ Riesgo } \\
\hline & & 1998-2003 & 2004-2009 & 2010-2015 & TS & \\
\hline SNC & CABA & $4,42(3,16-5,68)$ & $2,86(1,88-3,85)$ & $2,73(1,77-3,7)$ & $-0,038^{*}$ & $0,62^{*}$ \\
\hline (incluye defectos & Norte & $2,85(0,57-5,14)$ & $2,44(0,33-4,55)$ & $1,75(0,00-3,60)$ & $-0,034$ & 0,61 \\
\hline del cierre del & Centro & $3,98(2,18-5,79)$ & $2,37(1,03-3,71)$ & $2,41(1,11-3,71)$ & $-0,035$ & $0,61^{*}$ \\
\hline tubo neural) & Sur & $4,78(2,42-7,14)$ & $2,99(1,30-4,69)$ & $3,18(1,31-5,05)$ & $-0,041$ & 0,67 \\
\hline \multirow[t]{4}{*}{ Cardiovascular } & CABA & $11,35(9,34-13,37)$ & $8,66(6,95-10,37)$ & $8,53(6,83-10,24)$ & $-0,023^{*}$ & $0,75^{*}$ \\
\hline & Norte & $8,76(4,75-12,77)$ & $6,50(3,05-9,95)$ & $7,42(3,60-11,24)$ & $-0,020$ & 0,85 \\
\hline & Centro & $10,50(7,58-13,43)$ & $7,37(5,00-9,74)$ & $8,80(6,31-11,28)$ & $-0,020$ & 0,84 \\
\hline & Sur & $12,40(8,60-16,20)$ & $10,58(7,39-13,77)$ & $9,17(5,99-12,34)$ & $-0,016$ & $0,74^{*}$ \\
\hline \multirow{5}{*}{$\begin{array}{l}\text { Anomalías } \\
\text { cromosómicas }\end{array}$} & CABA & $2,31(1,40-3,22)$ & $1,43(0,73-2,13)$ & $3,45(2,37-4,54)$ & $0,039 *$ & $1,49^{*}$ \\
\hline & Norte & $2,45(0,32-4,56)$ & $1,42(0,00-3,03)$ & $3,49(0,87-6,11)$ & 0,044 & 1,43 \\
\hline & Centro & $2,26(0,91-3,62)$ & $1,52(0,45-2,60)$ & $3,35(1,82-4,88)$ & 0,034 & 1,48 \\
\hline & Sur & $2,45(0,77-1,14)$ & $0,86(0,00-1,76)$ & $3,54(1,57-5,52)$ & 0,043 & 1,44 \\
\hline & \multicolumn{6}{|c|}{ PM-MC } \\
\hline \multirow{4}{*}{$\begin{array}{l}\text { SNC } \\
\text { (incluye defectos } \\
\text { del cierre del } \\
\text { tubo neural) }\end{array}$} & CABA & $15,3(10,9-19,6)$ & $11,7(7,7-15,7)$ & $10,7(6,9-14,5)$ & $-0,028^{*}$ & $0,70^{*}$ \\
\hline & Norte & $11,4(2,3-20,5)$ & $12,5(1,7-23,3)$ & $8,1(0,0-16,7)$ & $-0,020$ & 0,71 \\
\hline & Centro & $15,1(8,3-21,9)$ & $11,1(4,8-17,4)$ & $10,5(4,8-16,1)$ & $-0,024$ & 0,70 \\
\hline & Sur & $15,8(8,0-23,6)$ & $10,5(4,5-16,4)$ & $11,2(4,6-17,7)$ & $-0,036$ & 0,71 \\
\hline \multirow[t]{4}{*}{ Cardiovascular } & CABA & $39,3(32,3-46,2)$ & $35,4(28,4-42,4)$ & $33,5(26,8-40,2)$ & $-0,013$ & 0,85 \\
\hline & Norte & $35,0(19,0-50,9)$ & $33,3(15,7-51,0)$ & $34,3(16,7-52,0)$ & $-0,006$ & 0,98 \\
\hline & Centro & $39,7(28,7-50,8)$ & $34,5(23,4-45,6)$ & $38,2(27,4-48,9)$ & $-0,010$ & 0,96 \\
\hline & Sur & $41,0(28,5-53,6)$ & $37,1(25,9-48,3)$ & $32,2(21,0-43,3)$ & $-0,013$ & 0,79 \\
\hline \multirow{4}{*}{$\begin{array}{l}\text { Anomalías } \\
\text { cromosómicas }\end{array}$} & CABA & $8,0(4,8-11,1)$ & $5,8(3,0-8,7)$ & $13,6(9,3-17,8)$ & $0,047^{*}$ & $1,70^{*}$ \\
\hline & Norte & $9,8(1,3-18,2)$ & $7,3(0,0-15,6)$ & $16,2(4,0-28,3)$ & 0,054 & 1,66 \\
\hline & Centro & $8,6(3,4-13,7)$ & $7,1(2,1-12,2)$ & $14,5(7,9-21,2)$ & $0,042^{*}$ & $1,70^{*}$ \\
\hline & Sur & $8,1(2,5-13,7)$ & $3,0(0,0-6,2)$ & $12,4(5,5-19,4)$ & 0,045 & 1,53 \\
\hline
\end{tabular}

* Nivel de significación $p<0,05$; IC 95: intervalo de confianza del $95 \%$; TMI-MC: tasa de mortalidad infantil por malformaciones congénitas; PM-MC: porcentaje de muertos por malformaciones congénitas; TS: tendencia secular; CABA: Ciudad Autónoma de Buenos Aires. 


\section{DISCUSIÓN}

No existen antecedentes sobre el análisis de la epidemiología de las muertes infantiles por
MC en grandes metrópolis. En Argentina, en el período 2002 y 2006, el 22,7 \% de las muertes infantiles se atribuyeron a MC; este porcentaje,

TABla 3. Tasa de mortalidad infantil por malformaciones congénitas específica, por malformaciones, tendencia secular y riesgo del último período (2010-2015) en relación con el basal (1998-2003) por subperíodos

\begin{tabular}{|c|c|c|c|c|c|c|}
\hline \multirow[t]{2}{*}{ Malformación } & \multirow[t]{2}{*}{$\begin{array}{c}\text { Código } \\
\text { CIE }\end{array}$} & \multicolumn{3}{|c|}{$\begin{array}{l}\text { TMI-MC (105) } \\
\text { (IC 95) }\end{array}$} & \multirow[t]{2}{*}{ TS } & \multirow[t]{2}{*}{ Riesgo } \\
\hline & & 1998-2003 & 2004-2009 & 2010-2015 & & \\
\hline Anencefalia & Q000 & $24,7(15,3-34,1)$ & $12,8(6,21-19,4)$ & $8,72(3,27-14,2)$ & $-0,085^{*}$ & $0,35^{*}$ \\
\hline Encefalocele & Q010-9 & $2,39(0,00-5,32)$ & $1,88(0,00-4,41)$ & $1,90(0,00-4,44)$ & $-0,001$ & 0,79 \\
\hline Microcefalia & Q020 & $1,20(0,00-3,26)$ & $1,13(0,00-3,09)$ & $0,00(0,00-0,00)$ & $-0,124$ & - \\
\hline Hidrocefalia & Q039 & $6,37(1,59-11,2)$ & $3,76(0,19-7,34)$ & $3,79(0,19-7,39)$ & $-0,041$ & 0,60 \\
\hline Holoprosencefalia & Q042 & $0,80(0,00-2,49)$ & $2,26(0,00-5,03)$ & $4,17(0,40-7,95)$ & $0,133^{*}$ & $5,23^{*}$ \\
\hline Hipoplasia del encéfalo & Q043 & $0,80(0,00-2,49)$ & $1,88(0,00-4,41)$ & $1,90(0,00-4,44)$ & 0,061 & 2,38 \\
\hline Espina bífida & Q050-9 & $2,39(0,00-5,32)$ & $0,75(0,00-2,35)$ & $0,76(0,00-2,37)$ & $-0,145^{*}$ & 0,38 \\
\hline Discordancia ventricular & Q203 & $1,99(0,00-4,66)$ & $2,64(0,00-5,62)$ & $1,14(0,00-3,11)$ & $-0,041$ & 0,57 \\
\hline Ventrículo de doble entrada & Q204 & $0,40(0,00-1,59)$ & $2,26(0,00-5,03)$ & $1,14(0,00-3,11)$ & 0,062 & 2,86 \\
\hline Defecto septal ventricular & Q210-12 & $0,80(0,00-2,49)$ & $0,38(0,00-1,51)$ & $0,76(0,00-2,37)$ & 0,000 & 0,95 \\
\hline Tetralogía de Fallot & Q213 & $1,99(0,00-4,66)$ & $0,75(0,00-2,35)$ & $1,14(0,00-3,11)$ & $-0,061$ & 0,57 \\
\hline Hipoplasia del VI & Q234 & $3,98(0,20-7,76)$ & $3,76(0,19-7,34)$ & $4,93(0,83-9,03)$ & 0,007 & 1,24 \\
\hline Conducto arterioso permeable & Q250 & $0,80(0,00-2,49)$ & $3,01(0,00-6,21)$ & $7,97(2,75-13,2)$ & $0,174^{*}$ & $9,99^{*}$ \\
\hline Coartación de la aorta & Q251 & $0,80(0,00-2,49)$ & $1,13(0,00-3,09)$ & $0,38(0,00-1,52)$ & $-0,010$ & 0,48 \\
\hline Hipoplasia pulmonar & Q336 & $13,9(6,87-21,0)$ & $16,19(8,78-23,6)$ & $7,97(2,75-13,2)$ & $-0,030$ & $0,57^{*}$ \\
\hline Agenesia renal & Q601-2 & $1,20(0,00-3,26)$ & $2,26(0,00-5,03)$ & $2,66(0,00-5,67)$ & 0,064 & 2,22 \\
\hline Síndrome de Potter & Q606 & $1,20(0,00-3,26)$ & $1,51(0,00-3,76)$ & $1,14(0,00-3,11)$ & $-0,007$ & 0,95 \\
\hline Poliquistosis renal & Q611-3 & $0,80(0,00-2,49)$ & $2,64(0,00-5,62)$ & $2,28(0,00-5,06)$ & 0,073 & 2,86 \\
\hline Displasia tanatofórica & Q771 & $0,00(0,00-0,00)$ & $0,38(0,00-1,51)$ & $1,14(0,00-3,11)$ & 0,307 & - \\
\hline Hernia diafragmática & Q790 & $13,9(6,87-21,0)$ & $12,1(5,66-18,4)$ & $12,1(5,70-18,6)$ & $-0,003$ & 0,87 \\
\hline Onfalocele & Q792 & $1,59(0,00-3,98)$ & $1,13(0,00-3,09)$ & $1,52(0,00-3,79)$ & 0,005 & 0,95 \\
\hline Gastrosquisis & Q793 & $2,39(0,00-5,32)$ & $3,39(0,00-6,78)$ & $4,93(0,83-9,03)$ & 0,064 & 2,06 \\
\hline Síndrome de Down & Q900-9 & $8,76(3,16-14,4)$ & $2,64(0,00-5,62)$ & $9,10(3,53-14,7)$ & 0,010 & 1,04 \\
\hline Síndrome de Edwards & Q911-3 & $9,56(3,71-15,4)$ & $7,53(2,48-12,6)$ & $16,3(8,85-23,8)$ & $0,048^{*}$ & $1,71^{*}$ \\
\hline Síndrome de Patau & Q914-7 & $3,19(0,00-6,57)$ & $4,14(0,40-7,89)$ & $6,45(1,76-11,1)$ & 0,042 & 2,02 \\
\hline
\end{tabular}

* Nivel de significación $p<0,05$; IC 95: intervalo de confianza del $95 \%$; TMI-MC: tasa de mortalidad infantil por malformaciones congénitas; TS: tendencia secular; VI: ventrículo izquierdo.

Figura 3. Gráfico de dispersión de la tasa de mortalidad infantil por malformaciones congénitas, del porcentaje de muertos por malformaciones congénitas y del primer componente principal de las variables socioeconómicas por comunas
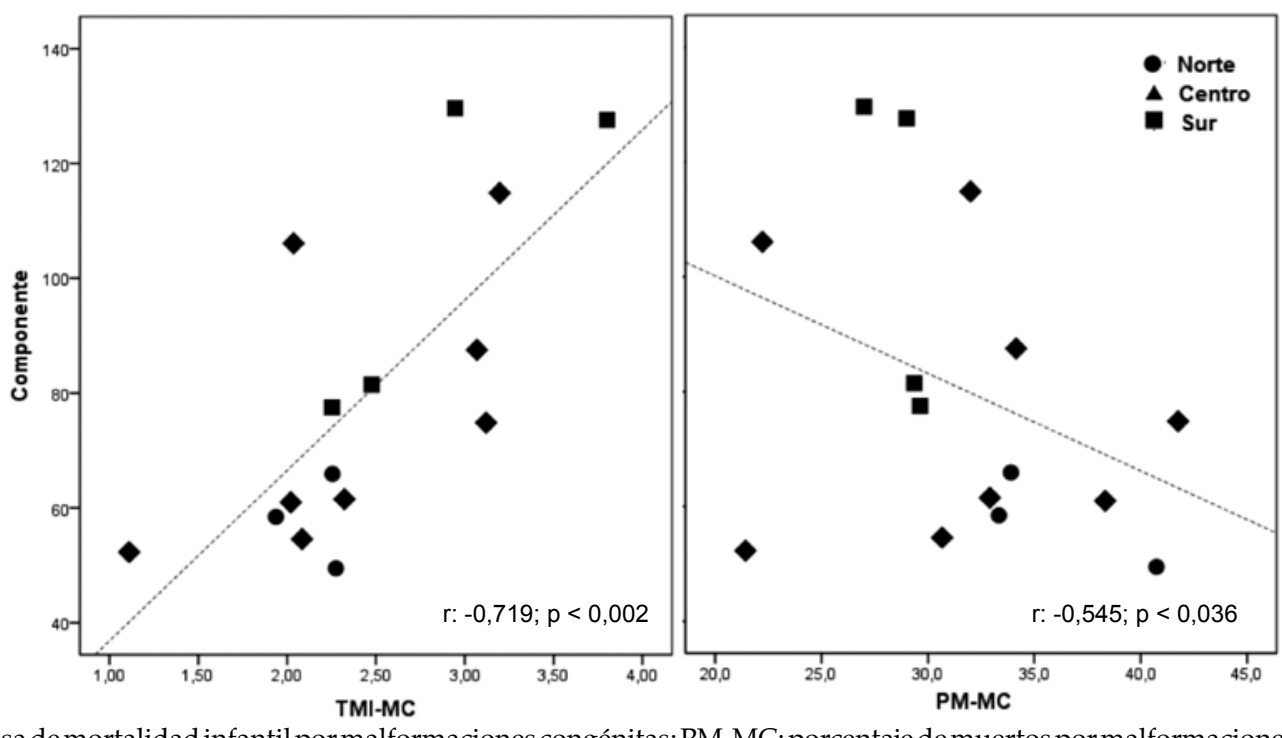

TMI-MC: tasa de mortalidad infantil por malformaciones congénitas; PM-MC: porcentaje de muertos por malformaciones congénitas. 
en la CABA, fue del $28,4 \%$ en el mismo período. ${ }^{6}$

Sintéticamente, lo que se observa es que todos los parámetros de muertes infantiles por MC analizados exhiben una heterogeneidad espacial y un comportamiento zonal diferencial a través del tiempo.

En Argentina, como en los países desarrollados ${ }^{2}$ y algunos países latinoamericanos, ${ }^{5}$ las muertes infantiles por $\mathrm{MC}$ presentan un patrón caracterizado por una TS negativa de la TMI-MC y una TS positiva del PM-MC. En el mismo sentido, se observa también en la CABA entre 1998 y 2015, donde, pese a las condiciones socioeconómicas más favorables respecto del resto del país, la TMI-MC desciende más sutilmente (el 0,9\%/ año), mientras el PM-MC aumenta (el 1,4 \% / año). Aunque las zonas geográficas de la CABA presentan también una TS negativa de la TMI-MC, pero estadísticamente no significativa, el menor descenso de este indicador se observa en la zona Sur. El PM-MC por zonas, como era de esperar de acuerdo con el patrón (TMI-MC con TS negativaPM-MC con TS positiva), presenta una TS positiva en todas las zonas, pero donde aumenta más significativamente es en la zona Sur, que exhibe el riesgo zonal con significación estadística más alto. No obstante, desde el punto de vista histórico, a través de todos los períodos, la TMI-MC más baja y el PM-MC más alto se presenta en la zona Norte; lo contrario se observa en la zona Sur. Igual situación se presenta cuando los indicadores de MI se analizan por años; los PM-MC más elevados se presentan en la zona Norte, y las TMI-MC más elevadas, en la zona Sur.

Dado que el peso de las muertes por MC recae en las malformaciones estructurales mayores, en especial, en los defectos del cierre del tubo neural y las malformaciones cardíacas, la TS negativa tanto de la TMI-MC como del PM-MC específica para estas malformaciones puede traducir indirectamente los esfuerzos realizados a nivel nacional y local para disminuir su prevalencia y tratar de manera precoz estos defectos congénitos. ${ }^{6}$ Para mejorar el diagnóstico prenatal y la cirugía neonatal temprana de las cardiopatías congénitas, existe una red de derivación y tratamiento oportuno integrada a través del Programa Nacional de Cardiopatías Congénitas. ${ }^{19}$ Además, en Argentina, el enriquecimiento obligatorio de la harina de trigo con ácido fólico se reglamentó a partir del año 2003. ${ }^{20}$ Por sistemas, se observa una TS negativa estadísticamente significativa para las malformaciones del SNC y del SCV, y, para las malformaciones específicas del SNC, históricamente, la TMI-MC y el PM-MC más elevados se observan, en general, en la zona Sur. Bronberg et al. (2011), estimaron una reducción del riesgo de mortalidad por anencefalia en Argentina, en el período 1998-2007, del $53 \%{ }^{21}$ En este, la tasa de mortalidad específica por anencefalia prefortificación a nivel nacional era de 3,10 (1998-2002) y, posfortificación, de 1,46 (2005-2007). En la CABA, las tasas en el período de prefortificación y posfortificación fueron menores, 2,65 y 1,30, respectivamente. De acuerdo con este trabajo, en el que se extiende el período de análisis (1998-2015), con datos provenientes de la misma fuente, la tasa de mortalidad específica por anencefalia continúa descendiendo, con una reducción significativa de las muertes infantiles por esta causa del $65 \%$.

Si bien, de modo global, en este estudio, se observa una disminución de la TS y del riesgo significativo del $25 \%$ de la mortalidad por defectos del SCV, la TS de las muertes infantiles específicas por ductus arterioso persistente (DAP) y su riesgo son significativamente positivos, lo cual podría atribuirse al limitado acceso a su tratamiento, a las complicaciones de la prematurez asociada a esta afección y al diagnóstico tardío. ${ }^{22}$

Los factores que condicionan el aumento significativo de la TMI-MC y del PM-MC debido a cromosomopatías son múltiples, interactúan entre sí y muestran una clara diferenciación social: posibilidad de acceso al diagnóstico prenatal, edad materna avanzada, calidad del diagnóstico prenatal de las cromosopatías, acceso al aborto electivo y características reproductivas según su lugar de residencia. Todos estos factores, sumados a la letalidad diferencial de las trisomías, explicarían el aumento de las muertes infantiles por cromosopatías. Si bien, en todas las zonas geográficas, la TS de la TMI-MC y del PM-MC debido a anomalías cromosómicas es positiva y estadísticamente no significativa, excepto para el PM-MC en la zona Centro, los valores más elevados de la TMI-MC y del PM-MC de las anomalías cromosómicas en el período 20102015 se presentan en las zonas Sur y Norte, respetivamente.

De acuerdo con el ACP, la CABA muestra una heterogeneidad del desarrollo socioeconómico por zonas geográficas verificada también por otras observaciones. ${ }^{9-15}$ La zona geográfica menos desarrollada de la CABA sería la zona Sur, donde, de manera concomitante, se presentan 
los valores más altos y más bajos de la TMI- MC y del PM-MC, respectivamente. Esto explicaría la correlación positiva de la TMI-MC y negativa del PM-MC con el indicador socioeconómico, que muestra un patrón distintivo que se caracteriza por un aumento de la TMI-MC con la disminución del desarrollo y lo contrario con el PM-MC. Este patrón ha llevado a utilizar el PM-MC como un proxy socioeconómico, lo cual ha sido verificado previamente en Chile, ${ }^{5}$ en Argentina ${ }^{7}$ y en 36 países de Europa, de Oriente Medio, de América, de Asia y del Pacífico. ${ }^{2}$

La estructura socioespacial y sociohabitacional de la CABA es producto de un largo desarrollo histórico, en el cual factores socioeconómicos, políticos y territoriales, tanto a nivel metropolitano como nacional, fueron modificando las condiciones de acceso al suelo y a la vivienda en este ámbito. Las diferencias y desigualdades metropolitanas, por la dimensión espacial de los procesos sociourbanos, constituyen una temática compleja y atravesada por profundas discusiones conceptuales y metodológicas. ${ }^{11}$ Las heterogeneidades espaciales socioeconómicas de la CABA se traducirían en diferencias espaciales en salud, como lo indica la epidemiología de las MI por MC analizada en este trabajo.

\section{CONCLUSIÓN}

La CABA, pese a presentar, entre los años 1998 y 2015, un estadio avanzado de la transición epidemiológica de las causas de MI, concomitantemente, exhibe una gran heterogeneidad de los indicadores de MI por MC, que coincide con la diversidad socioeconómica de sus zonas geográficas.

\section{REFERENCIAS}

1. WHO. Special Topic: The ten leading causes of death in countries of the Americas. In: Health Stastitics from the Americas. 2006. [Consulta: 10 de julio de 2018]. Disponible en: http:/ / www.paho.org/English/DD/AIS/HSA2006_ ST.pdf.

2. Rosano A, Botto LD, Botting B, Mastroiacovo P. Infant mortality and congenital anomalies from 1950 to 1994: an international perspective. J Epidemiol Community Health. 2000; 54(9):660-6.

3. Copeland G, Kirby RS. Using birth defects registry data to evaluate infant and childhood mortality associated with birth defects: an alternative to traditional mortality assessment using underlying cause of death statistics. Birth Defects Res A Clin Mol Teratol. 2007; 79(11):792-7.

4. Szot J. Mortalidad infantil por malformaciones congénitas: Chile, 1985-2001. Rev Chil Pediatr. 2004; 75(4):347-54.
5. Dipierri JE, Acevedo NE, Bronberg RA. Mortalidad infantil por malformaciones congénitas en Chile: análisis temporal y espacial, 1997-2011. Rev Panam Salud Pública. 2015; 38(5):380-7.

6. Bronberg R, Alfaro E, Chaves E, Dipierri J. Mortalidad infantil por malformaciones congénitas en Argentina: análisis del quinquenio 2002-2006. Arch Argent Pediatr. 2009; 7(3):203-11.

7. Bronberg R, Gutiérrez Redomero E, Alonso M, Dipierri J. Mortalidad infantil por malformaciones congénitas y condición socioeconómica: el caso de la Argentina. Rev Panam Salud Pública. 2012; 31(6):469-75.

8. Dirección General de Estadística y Censos (Ministerio de Hacienda GCBA). Anuario Estadístico 2015 Ciudad de Buenos Aires. [Consulta: 10 de julio de 2018]. Disponible en: http: / / www.estadisticaciudad.gob.ar/ eyc / ?p=61948.

9. Marcos M, Mera G. La dimensión espacial de las diferencias sociales: Alcances y limitaciones metodológicoconceptuales de las herramientas estadísticas para abordar la distribución espacial intraurbana. Rev Univ Geogr. 2011; 20(1):41-65.

10. ManzanoFA, VelázquezGA. Calidad de viday desigualdad en el área metropolitana de Buenos Aires. Análisis a nivel de fracción censal (2010). Cardinalis (Córdoba En línea). 2016; 4(6):19-44.

11. Di Virgilio MM, Marcos M, Mera G. Las ciudades dentro de la ciudad: características sociodemográficas y habitacionales de la Ciudad de Buenos Aires según sus tipos de hábitat. Poblac B Aires. 2015; 12(22):33-57.

12. Bronberg RA, Dipierri JE, Alfaro EL, Barrai I, et al. Isonymy structure of Buenos Aires city. Hum Biol. 2009; 81(4):447-61.

13. Usach N, Garrido-YserteR, Gallo-Rivera MT. Organización territorial y funcional de la metrópoli de Buenos Aires. EURE (Santiago). 2017; 43(128):55-80.

14. Grushka C, Baum D, Sanni L. Vivir y morir en las comunas de la Ciudad de Buenos Aires: un estudio de diferenciales. Poblac B Aires. 2013; 10(18):33-44.

15. Mazzeo V.La mortalidad dela primera infancia en la Ciudad de Buenos Aires en el período 1860-2002. Pap Poblac. 2007; 13(53):241-72.

16. Dirección de Estadística e Información deSalud. Ministerio de Salud y Desarrollo Social. Indicadores Básicos. [Acceso: 10 dejulio de 2018]. Disponible en: http: / / www.deis.msal. gov.ar/index.php/indicadores-basicos/.

17. Organización Panamericana de la Salud. Clasificación Internacional de Enfermedades y problemas relacionados con la salud. 10. ${ }^{\mathrm{a}}$ rev.; vol. 2. Washington, DC: OPS; 1995. [Acceso: 10 de julio de 2018]. Disponible en: ais.paho.org/ classifications/Chapters/pdf/Volume2.pdf.

18. DirecciónGeneraldeEstadísticayCensosdelaCABA.Anuario estadístico 2016. [Acceso: 10 de julio de 2018]. Disponible en: http: / / www.estadisticaciudad.gob.ar/eyc/wp-content / uploads/2017/10/anuario_estadistico_2016.pdf.

19. Chernovesky G. Programa nacional de cardiopatías congénitas. Rev Hosp Mat Inf Ramón Sardá. 2011; 30(1):38-41.

20. Calvo E. Fortificación con ácido fólico y defectos del tubo neural. Arch Argent Pediatr. 2008; 106(4):291-2.

21. Bronberg R, Alfaro E, Chaves E, Andrade A, et al. Mortalidad infantil por anencefalia en la Argentina. Análisis espacial y temporal (1998-2007). Arch Argent Pediatr. 2011; 109(2):117-23.

22. Golombek SG, Sola A, Baquero H, Borbonet D, et al. Primer consenso clínico de SIBEN: enfoque diagnóstico y terapéutico del ductus arterioso permeable en recién nacidos pretérmino. An Pediatr (Barc). 2008; 69(5):454-81. 